\title{
Synthesis of a Crystal Violet-Cadmium Hydroxyquinoline Iodine Nanocomposite for the Photoelectrochemical Sensing of Ascorbic Acid
}

\author{
Shangwang Le $e^{1}$ Qian Jiang ${ }^{1}$, Hongcheng Pan ${ }^{1,2,3, *}$ \\ ${ }^{1}$ College of Chemistry and Bioengineering, Guilin University of Technology, 12 Jiangan Road, Guilin \\ 541004, P. R. China. \\ ${ }^{2}$ Guangxi College and University Key Laboratory of Food Safety and Detection, Guilin University of \\ Technology, 12 Jiangan Road, Guilin 541004, P. R. China. \\ ${ }^{3}$ Guangxi Key Laboratory of Electrochemistry and Magnetochemistry Functional Materials, Guilin \\ University of Technology, 12 Jiangan Road, Guilin 541004, P. R. China. \\ *E-mail: hcpan@163.com
}

doi: $10.20964 / 2018.09 .76$

Received: 24 April 2018 / Accepted: 18 July 2018 / Published: 5 August 2018

\begin{abstract}
A crystal violet-cadmium hydroxyquinoline iodine (CV-CdqI) nanocomposite was synthesized by adsorbing crystal violet $(\mathrm{CV})$ onto cadmium hydroxyquinoline iodine (CdqI) nanowires. The CdqI nanowires were synthesized by mixing $100 \mathrm{~mL}$ of an aqueous $0.03 \mathrm{M} \mathrm{CdI}_{2}$ solution with $100 \mathrm{~mL}$ of a $0.01 \mathrm{M}$ 8-hydroxyquinoline ethanol solution in an ultrasonic bath. Scanning electron microscopy showed that CdqI exhibited a regular nanorod morphology with diameters of 30-60 nm and lengths of 400-600 nm. After adsorbing crystal violet, the CdqI nanowires become aggregated to form the CVCdqI nanocomposite. X-ray powder diffraction (XRD) revealed that the XRD patterns of the CdqI nanowires and CV-CdqI nanocomposite are essentially the same, and no new phase was generated in the XRD pattern after the adsorption of CV. The fluorescence and photoelectrochemical (PEC) properties of the CV-CdqI nanocomposite were investigated. The CV-CdqI nanocomposite emits a yellow-green fluorescence with a maximum emission at $575 \mathrm{~nm}$, which is similar to that of the CdqI nanowires. PEC measurements showed that the photocurrent response of the CV-CdqI nanocomposite spin-coated FTO electrode was stable and repeatable under the periodic on/off illumination of a $300 \mathrm{~W}$ xenon lamp at $0 \mathrm{~V}$ bias. Furthermore, the $\mathrm{CV}-\mathrm{CdqI} / \mathrm{FTO}$ electrode was used to construct a sensor for the PEC detection of ascorbic acid (AA). In the range of 1.4 to $10 \mu \mathrm{M}$, the photocurrent of the CV$\mathrm{Cdq} / \mathrm{FTO}$ electrode was inversely proportional to the concentration of AA with a detection limit of 0.2 $\mu \mathrm{M}$. The method was used for the determination of AA in oranges (varied from 32.1 to $50.8 \mathrm{mg} / 100 \mathrm{~g}$ fresh weight).
\end{abstract}

Keywords: Crystal violet; cadmium hydroxyquinoline iodine; nanocomposite; photochemical sensing; ascorbic acid 


\section{$\underline{\text { FULL TEXT }}$}

(C) 2018 The Authors. Published by ESG (www.electrochemsci.org). This article is an open access article distributed under the terms and conditions of the Creative Commons Attribution license (http://creativecommons.org/licenses/by/4.0/). 